\title{
A Meta-Analysis of the Neuropsychological Sequelae of Chemotherapy-Only Treatment for Pediatric Acute Lymphoblastic Leukemia
}

\author{
Catherine C. Peterson, $\mathrm{PhD}^{1}{ }^{1}$ Courtney E. Johnson, $\mathrm{MA}^{2}$ Lisa Y. Ramirez, $\mathrm{MA}^{2}$ \\ Samantha Huestis, $\mathrm{MA}^{2}{ }^{2}$ Ahna L.H. Pai, $\mathrm{PhD}^{3}{ }^{3}$ Heath A. Demaree, $\mathrm{PhD},{ }^{2}$ \\ and Dennis Drotar, $\mathrm{PhD}^{3}$
}

Background. Mixed findings on the neuropsychological sequelae of chemotherapy-only treatment for pediatric acute lymphoblastic leukemia (ALL), without radiation, indicate the need for a comprehensive meta-analytic review. The purpose of the current study was to conduct a meta-analysis assessing neuropsychological and academic functioning differences between children with ALL treated solely with chemotherapy and comparison groups. Procedures. Thirteen articles met inclusion criteria for the metaanalysis and were analyzed using a random effects model, weighted least squares methods. Results. Mean effect sizes were significantly different from zero for multiple domains of intelligence and academic achievement; processing speed; verbal memory; and some aspects of executive functioning and fine motor skills, indicating worse functioning in ALL survivors. Effect sizes for visualmotor skills and visual memory were not significantly different from zero. Conclusions. Results support the presence of neuropsychological and academic sequelae for ALL survivors treated solely with chemotherapy and highlight the need for ongoing follow-up of children with ALL using a standardized neuropsychological test battery and research methodology. Pediatr Blood Cancer 2008;51:99-104. (C) 2008 Wiley-Liss, Inc.

Key words: chemotherapy; leukemia; meta-analysis; neuropsychological; pediatric cancer survivor

\section{INTRODUCTION}

Research examining the neuropsychological outcomes of whole brain radiation therapy (RT) plus intrathecal (IT) chemotherapy for acute lymphoblastic leukemia (ALL) has indicated declines in nonverbal intelligence, math achievement, visual-motor integration, processing speed, attention, executive functioning, and memory [1-6]. Two meta-analytic reviews of the neuropsychological outcomes of RT [1,7] documented significantly decreased intellectual functioning in ALL survivors, as well as poorer academic achievement, attention, information processing, executive functioning, psychomotor and visuospatial skills, and memory compared to controls. Both meta-analytic reviews included children who had received RT; however, the outcomes of chemotherapy-only treatment for ALL have not been subjected to meta-analytic review. Campbell and colleagues [7] noted that their meta-analysis could not conclusively determine the impact of chemotherapy-only treatment. Thus, it is now important to describe, using metaanalytic techniques, the long-term neuropsychological sequelae of chemotherapy-only for pediatric leukemia.

Research suggests that the underlying basis for neuropsychological deficits may be the impact of radiation on white matter density, by which impaired myelinization affects nondominant hemisphere functions and slowed cortical activity [8,9]. Although treatment protocols were modified so that few ALL patients receive RT, most ALL patients still receive IT chemotherapy (particularly methotrexate $[\mathrm{MTX}]$ ), often combined with intravenous or oral chemotherapy, resulting in high doses of systemic and central nervous system (CNS)-targeted chemotherapeutic agents during critical brain development. It has been reported that IT MTX, even without RT, may be linked to white matter changes, calcifications, leukoencephalopathy, cortical atrophy, and seizures [10].

One review of neuropsychological outcomes of CNS chemotherapy concluded that two-thirds of studies indicated decreased intellectual functioning in ALL survivors receiving chemotherapy compared to controls [10]. Numerous empirical studies of neuropsychological outcomes in ALL survivors have indicated deficits in performance IQ (PIQ) [11-14], academic achievement [13,15,16], and specific cognitive skills including processing speed, attention, visual-spatial skills, fine motor skills, and nonverbal memory [12,17-20]. Some studies, however, report only slight or no impairment $[21,22]$. To reconcile these mixed findings, and given evidence of neuropsychological dysfunction associated with CNS treatment for ALL [10], it is critical to synthesize available data using effect size statistics to estimate the impact of chemotherapy on intellectual, neuropsychological, and academic outcomes.

\section{METHOD}

\section{Article Identification}

We conducted literature searches using MEDLINE and PsycInfo databases and reference sections of relevant articles; additional details of search terms and inclusion/exclusion criteria can be found in the Supplementary materials. Following identification of relevant articles, each article was examined in detail by the authors to

This article contains Supplementary Material available at http:// www.interscience.wiley.com/jpages/1545-5009/suppmat.

${ }^{1}$ Division of Child Behavioral Health, Department of Pediatrics and Communicable Diseases, University of Michigan Medical School, Michigan; ${ }^{2}$ Department of Psychology, Case Western Reserve University, Ohio; ${ }^{3}$ Division of Behavioral Medicine and Clinical Psychology, Cincinnati Children's Hospital Medical Center, Ohio

Grant sponsor: Lance Armstrong Foundation.

*Correspondence to: Catherine C. Peterson, Division of Child Behavioral Health, University of Michigan Medical School, 1500 East Medical Center Drive, 1924 Taubman Center, Ann Arbor, MI 48109. E-mail: catpeter@med.umich.edu

Received 27 August 2007; Accepted 17 January 2008 
determine that they included participants who had completed chemotherapy-only treatment for pediatric ALL and a comparison group that did not receive CNS treatment. Articles had to be in English and include original empirical data sufficient to calculate effect sizes (i.e., means, standard deviations, and sample sizes for ALL and control groups). From the 160 articles, studies were excluded due to sample characteristics (i.e., did not examine a homogeneous ALL survivor sample or did not have an eligible comparison group; 40\%), were published prior to 1990 (to focus on recent treatment protocols that administered CNS chemotherapy only; $25 \%$ ), did not report eligible neuropsychological data (11\%), or did not report any empirical data $(9 \%)$. Of the 21 remaining articles, eight were excluded due to unusable data (e.g., did not report all necessary data; measures were not comparable to measures in any other study).

For 13 articles meeting inclusion criteria [11-14,16-18,2025], the following variables were abstracted: sample size, gender, mean age, mean age at diagnosis, ethnicity, SES, medical diagnoses, treatment modalities, time since diagnosis, and means and standard deviations for cognitive or academic outcomes. A neuropsychologist verified that study measures were established neuropsychological measures (i.e., if cited in the Compendium of Neuropsychological Tests [26]) versus an investigator's own measure and classified variables into constructs. See Table I for a summary of the 13 articles and sample demographics.

\section{Effect Size Calculations}

Effect sizes were calculated using ZumaStat software [27] to compare ALL and comparison groups on outcome measures. Differences between control group means and ALL group means were divided by the pooled group standard deviations, yielding a Cohen's d for each construct [28]. Random effects models, weighted least squares methods, were employed for primary analyses [29]. A positive effect size indicated better performance in the control group. Cohen's classification was used to interpret effect sizes, where a mean effect size of $\mathrm{M}=0.20$ is considered a small effect, $\mathrm{M}=0.50$ is medium, and $\mathrm{M}=0.80$ is large.

\section{Tests of Homogeneity $\left(\mathrm{Q}_{\mathrm{T}}\right)$}

The test of $\mathrm{Q}_{\mathrm{T}}$ indicates the internal consistency of study outcome groupings. A significant $\mathrm{Q}_{\mathrm{T}}$ indicates that variability in the sample is greater than expected from sampling error alone and the data should be examined for moderating factors [30]. When Q statistics were significant, effect sizes were re-calculated excluding studies that used test normative data for their comparison group, as those samples were significantly larger than recruited control groups. If the $\mathrm{Q}_{\mathrm{T}}$ was still significant, effect sizes were re-calculated excluding studies that used translations of tests, due to the potential for a translated test not to be comparable to the original version, thereby creating increased variance. Test norms and translations were selected for removal because they potentially decrease neuropsychological assessment reliability across studies.

\section{Gender Analyses}

Based on literature indicating increased neuropsychological sequelae for girls with ALL [31], post hoc analyses of variance (ANOVAs) were calculated if effect sizes were heterogeneous based on the Q statistic. Two studies were identified that reported sufficient data separated by gender.

\section{RESULTS}

The overall ALL sample contained a mean of 27 participants per study ( $\mathrm{M}$ age $=5.3$ years at diagnosis; $\mathrm{M}$ time since treatment $=4.7$ years; $M$ age $=11.1$ years at assessment). Gender breakdown was 13.6 females and 14.4 males per study. Comparison groups (excluding studies that used test norm groups for comparison groups) had a mean of 26 participants per study ( $\mathrm{M}$ age $=12.0$ years at assessment). Gender breakdown of comparison groups was 13.6 females and 14.7 males per study. Nine studies reported information regarding participant ethnicities, which was predominantly Caucasian. Specific chemotherapy protocols were described in nine studies, and all included IT chemotherapy; seven of the nine specified that patients received triple IT (TIT) chemotherapy (MTX, cytosine arabinoside, and hydrocortisone). All studies were crosssectional.

Mean effect sizes were calculated for full scale intelligence (FSIQ), verbal IQ (VIQ), PIQ, math achievement, reading achievement, freedom from distractibility index, perceptual organization index, coding, digit span, finger tapping, Purdue pegboard (both hands), Purdue pegboard (preferred hand), trails B, and verbal memory. Table II presents weighted mean effect sizes, confidence intervals, and Q statistics for all constructs. With one exception (finger tapping), significant group differences indicated poorer functioning in the ALL group.

\section{Intelligence}

Mean effect sizes for FSIQ were significantly different from zero $(\mathrm{M}=0.55,95 \% \mathrm{CI}=.27-0.83, \mathrm{n}=10)$, indicating that children with ALL had significantly lower FSIQ scores relative to control groups. The $\mathrm{Q}$ statistic for $\mathrm{Q}_{\mathrm{T}}$ was significant $\left(\mathrm{Q}_{\mathrm{T}}=22.85\right.$, $P<0.01)$. Therefore, mean effect sizes were recalculated by eliminating three studies that utilized test norms as a comparison group. The resulting effect size was larger $(\mathrm{M}=0.76,95 \%$ $\mathrm{CI}=0.26-1.26, \mathrm{n}=7$ ), but the $\mathrm{Q}$ still indicated heterogeneity $\left(\mathrm{Q}_{\mathrm{T}}=22, P<0.01\right)$. Next, the three studies utilizing foreign translations of the measure were excluded; the recalculated mean effect size was still significantly different from zero $(\mathrm{M}=0.76,95 \%$ $\mathrm{CI}=0.42-1.12, \mathrm{n}=7$ ), and the $\mathrm{Q}$ was not significant. Results suggest that ALL survivors demonstrated significantly lower IQ scores than controls.

Similar results were found for index scores of the Wechsler intelligence measures [32-35]. Children with ALL had significantly lower VIQ and PIQ scores, with a medium mean effect size significantly different from zero. The Q statistic was significant for VIQ and PIQ and remained significant excluding norms. The freedom from distractibility index and perceptual organization index scores were significantly different from zero (Q statistics not significant), indicating lower scores in the ALL group. The verbal comprehension index did not significantly differ between groups $(\mathrm{n}=2)$.

Subtest-level findings were inconsistent. Effect sizes were significantly different from zero for digit span and coding but not for arithmetic, block design, similarities, or vocabulary. Q statistics were significant for arithmetic, block design, and coding; when 
TABLE I. Summary of Demographic Characteristics From Articles Included in Meta-Analysis

\begin{tabular}{|c|c|c|c|c|c|c|c|c|c|}
\hline \multirow[b]{2}{*}{ Source } & \multicolumn{5}{|c|}{ ALL Survivors } & \multicolumn{4}{|c|}{ Controls } \\
\hline & $\mathrm{N}$ & $\begin{array}{l}\text { Mean age } \\
\text { (years) at } \\
\text { diagnosis }\end{array}$ & $\begin{array}{l}\text { Mean time } \\
\text { (years) since } \\
\text { treatment }\end{array}$ & $\begin{array}{l}\text { Mean age } \\
\text { (years) at } \\
\text { evaluation }\end{array}$ & $\%$ Female & $\begin{array}{c}\text { Group } \\
\text { type }\end{array}$ & $\mathrm{N}$ & $\begin{array}{l}\text { Mean age } \\
\text { (years) at } \\
\text { evaluation }\end{array}$ & $\%$ Female \\
\hline Kaemingk et al. [16] & 15 & 5.44 & 4.4 & 12.62 & 40 & $a, b$ & 15 & 11.97 & 40 \\
\hline Schatz et al. [20] & 8 & 7 & 6.7 & 13.9 & 75 & $a, b$ & 24 & 17.4 & 58 \\
\hline Brown et al. [25] & 11 & NR & 1 & 7.55 & 64 & $\mathrm{a}$ & 12 & 9.02 & 50 \\
\hline Hill et al. [17] & 10 & $1-5$ years & $\geq 3$ & 10.3 & NR & $a, b$ & 10 & 10.1 & NR \\
\hline Lesnick et al. [18] & 10 & $1-5$ years & $\geq 3$ & $6.9-13.5$ & 60 & $a, b$ & 10 & $6.0-13.0$ & 60 \\
\hline Raymond-Speden et al. [13] & 21 & 4.1 & $\overline{4} .9$ & 9.4 & 43 & $\mathrm{~b}$ & 21 & 10.6 & 48 \\
\hline Giralt et al. [12] & 29 & 5.48 & $\geq 3$ & 11.37 & 48 & $\mathrm{a}, \mathrm{c}$ & 46 & 11.46 & 37 \\
\hline von der Weid et al. [14] & 132 & $4.8^{*}$ & $\geq 2$ & $14.7 *$ & 50 & $\mathrm{c}$ & 100 & $15.8^{*}$ & 46 \\
\hline Rodgers et al. [21] & 17 & 4 & $\overline{5}$ & 9.72 & 35 & a & 17 & 11.5 & 59 \\
\hline Kingma et al. [23] & 17 & $3.5^{*}$ & $\geq 5$ & $9.3^{*}$ & 41 & $\mathrm{~d}$ & 225 & NR & NR \\
\hline Kingma et al. [22] & 20 & $3.4 *$ & $\bar{N} R$ & $10.3 *$ & 45 & $\mathrm{~d}$ & 225 & NR & NR \\
\hline Schatz et al. [24] & 9 & 6.9 & 6.9 & 13.9 & 78 & $a, b$ & 9 & 14.3 & 78 \\
\hline Brown et al. [11] & $20-43$ & 4.3 & 3.8 & 11 & 43 & $\mathrm{~d}$ & $* *$ & NR & NR \\
\hline
\end{tabular}

ALL, acute lymphoblastic leukemia; NR, not reported. Control group types: a, healthy, family member (e.g., sibling); b, healthy, non-family (e.g., friend, neighbor, classmate); c, cancer patient, non-CNS tumor; d, test normative sample; *reported median versus mean for age variables; therefore, these studies were excluded from mean age calculations of overall sample; **control N varied depending on test used: Wechsler Intelligence Scale for Children (WISC) normative sample =2,200; Woodcock-Johnson (WJ) normative sample = 3,245; Beery Test of Visual-Motor Integration (VMI) normative sample $=2,734$.

arithmetic and coding were re-analyzed excluding translations, the recalculated arithmetic mean effect size was not significant, whereas the coding recalculated mean effect size was significant.

\section{Academic Achievement}

Effect sizes were significantly different from zero for math achievement and reading achievement. Results suggest that ALL survivors demonstrated significantly lower academic achievement than comparison groups on both achievement domains.

\section{Neuropsychological Constructs}

Results were inconsistent for measures of visual-motor integration, fine motor skills, and reaction time. Effect sizes were significantly different from zero on the Purdue pegboard task for both hands and preferred hand but not for Assembly. Q statistics were not significant. Effect sizes for VMI were not significant. Effect sizes were significantly different from zero for the finger tapping test, but effect sizes were negative, indicating that the ALL group scored higher on this test than the comparison groups. Children with ALL performed significantly worse on Trails B (a measure of executive functioning) but not Trails A (a measure of fine motor tracking). Verbal fluency comparison was not significant. The effect size for verbal memory was significantly different from zero, indicating that children with ALL performed more poorly than controls. Visual memory effect sizes were not significant.

\section{Gender Comparisons}

Post hoc ANOVAs on IQ constructs were conducted to explore potential gender differences [31]. Mean effect sizes were signifi- cantly different from zero for FSIQ $(\mathrm{M}=-0.57,95 \% \mathrm{CI}=-0.86$ $-0.28, \mathrm{n}=2)$, PIQ $(\mathrm{M}=-0.49,95 \% \mathrm{CI}=-0.78--0.19, \mathrm{n}=2)$, and VIQ $(\mathrm{M}=-0.51,95 \% \mathrm{CI}=-0.80--0.21, \mathrm{n}=2)$, indicating that girls performed worse than boys.

\section{DISCUSSION}

These findings present empirical support, using effect size statistics, for a pattern of neuropsychological sequelae of modern chemotherapy-only treatment protocols. These effect size data support research documenting neuropsychological late effects of childhood cancer, particularly given recent reports of no impairment or only mild impairment on select outcome measures following chemotherapy [22]. Results suggest that intellectual functioning does appear to be affected in ALL patients, even without RT, particularly in the areas of perceptual reasoning skills, working memory, and processing speed. Verbal subtests, however, were not significantly different between the groups, suggesting that select verbal skills may be spared in ALL survivors. This pattern of strengths and weaknesses is consistent with previously reviewed evidence [10], but this meta-analytic review provides synthesis of effect sizes from multiple studies supporting this pattern of strengths and weaknesses in ALL survivors.

Findings indicated that ALL survivors exhibit difficulty attaining academic progress in both math and reading. Although previous research has focused on math achievement [16], these data suggest that reading achievement also may be affected. Neuropsychological findings were mixed, with some evidence of fine motor, executive function, and verbal memory weaknesses in ALL survivors. These results, however, were based on very small samples and therefore, should be interpreted very cautiously. Post hoc analyses examining gender differences on intelligence tests also were based on small samples, so these findings must be interpreted with extreme caution. Nonetheless, our finding was consistent with reports that girls may 
TABLE II. Weighted Mean Effect Sizes; 95\% Confidence Intervals; and Q Statistics for Neuropsychological Constructs

\begin{tabular}{|c|c|c|c|c|}
\hline Measure/construct & $\mathrm{N}$ of studies & $\begin{array}{l}\text { Weighted } \\
\text { M ES }\end{array}$ & $95 \% \mathrm{CI}$ & Q \\
\hline General intelligence & 10 & $0.55^{* *}$ & $0.27-0.83$ & $22.85 * *$ \\
\hline Excluding normative data ${ }^{\dagger}$ & 7 & $0.76^{* *}$ & $0.26-1.26$ & $22 * *$ \\
\hline Excluding translations ${ }^{\dagger}$ & 7 & $0.76^{* *}$ & $0.42-1.12$ & 10.72 \\
\hline Verbal IQ & 6 & $0.46^{* *}$ & $0.11-0.81$ & $18.06 * *$ \\
\hline Excluding normative data & 3 & 0.86 & $0.09-1.81$ & $14.90 * *$ \\
\hline Excluding translations & 3 & 0.87 & $0.06-1.80$ & $13.59 * *$ \\
\hline Performance IQ & 6 & $0.42 *$ & $0.03-0.81$ & $23.58 * *$ \\
\hline Excluding normative data & 3 & 0.64 & $0.27-1.55$ & $14.30 * *$ \\
\hline Excluding translations & 3 & $0.73 *$ & $0.14-1.32$ & 5.78 \\
\hline Freedom from distractibility index & 2 & $0.54 * *$ & $0.25-0.83$ & 0.13 \\
\hline Perceptual organization index & 2 & $0.70 * *$ & $0.40-0.99$ & 0.96 \\
\hline Verbal comprehension index & 2 & 0.48 & $0.38-1.33$ & $5.73 *$ \\
\hline Arithmetic (Wechsler subtest) & 3 & 0.40 & $0.19-0.99$ & $6.75^{*}$ \\
\hline Excluding translations & 2 & 0.66 & $0.26-1.58$ & 3.23 \\
\hline Block design (Wechsler subtest) & 2 & 0.27 & $0.34-0.88$ & $4.07 *$ \\
\hline Coding (Wechsler subtest) & 4 & 0.48 & $0.02-0.98$ & $9.73^{*}$ \\
\hline Excluding translations & 3 & $0.70 * *$ & $0.27-1.12$ & 2.28 \\
\hline Digit span (Wechsler subtest) & 6 & $0.28 *$ & $0.04-0.52$ & 7.35 \\
\hline Similarities (Wechsler subtest) & 2 & 0.34 & $0.14-0.81$ & 2.56 \\
\hline Vocabulary (Wechsler subtest) & 2 & 0.27 & $0.16-0.70$ & 2.19 \\
\hline Math achievement & 5 & $0.61 * *$ & $0.20-1.03$ & 7.88 \\
\hline Reading achievement & 5 & $0.65^{*}$ & $0.03-1.27$ & $17.54 * *$ \\
\hline Excluding normative data & 4 & $0.87 * *$ & $0.48-1.26$ & 2.31 \\
\hline VMI & 4 & 0.37 & $0.19-0.93$ & $13.95 * *$ \\
\hline Finger tapping (preferred hand) & 2 & $-0.35^{*}$ & $-0.68--0.01$ & 0.94 \\
\hline Purdue pegboard (assembly) & 2 & -0.11 & $-0.45-0.22$ & 0.09 \\
\hline Purdue pegboard (both hands) & 2 & $0.38 *$ & $0.04-0.71$ & 0.04 \\
\hline Purdue pegboard (preferred hand) & 3 & $0.39 *$ & $0.08-0.69$ & 1.94 \\
\hline Verbal fluency & 2 & 0.41 & $0.06-0.88$ & 0.86 \\
\hline Trails A & 3 & 0.32 & $-0.24-0.87$ & 4.65 \\
\hline Trails B & 3 & $0.70 * *$ & $0.18-1.23$ & 3.97 \\
\hline Verbal memory & 2 & $1.16^{* *}$ & $0.54-1.79$ & 1.06 \\
\hline Visual memory & 2 & 1.03 & $0.64-2.70$ & $6.84 * *$ \\
\hline
\end{tabular}

ES, effect size; CI, confidence interval; IQ, intelligence quotient; VMI, Beery test of visual-motor integration; $* P<0.05 ; * * P<0.01$; ${ }^{\dagger}$ when the $\mathrm{Q}$ statistic indicated significant heterogeneity, analyses were re-run excluding either: (a) studies that used normative data as their comparison group versus a recruited control sample, or (b) studies that used translated versions of tests originally created in English, in order to reduce the potential for heterogeneity whenever possible. All data that could be calculated are presented.

be at greater risk for neuropsychological late effects than boys [31], warranting further investigation into gender differences in neuropsychological development.

The limitations of the meta-analysis reflect the state of the literature, particularly the limited number of studies that could be included due to methodological variability. We could not perform post hoc ANOVAs on potential risk factors of age at diagnosis and time since diagnosis, as few studies presented data separated into groups by age at diagnosis or time since diagnosis. Further, several studies were excluded due to the use of translated or newly developed measures that were not comparable to established neuropsychological measures. Other studies used heterogeneous samples such as a cancer sample that included another leukemia subtype or lymphoma. We focused on a homogeneous ALL sample, despite the loss of usable data, to facilitate more precise understanding of neuropsychological sequelae of a specific treatment component for ALL. A meta-analysis also can be limited by the impact of publication bias, which may attenuate the strength of effects found. It was not possible to illustrate publication bias for visual examination, however, as the number of studies was far too small for accurate interpretation [30].

These findings have bearing on future research and clinical practice in the management of neuropsychological sequelae of pediatric ALL. Treatment intensity may be an important moderator of outcomes, as multiple IT agents (e.g., TIT chemotherapy) may impact neuropsychological sequelae. It has been proposed that IT cytosine arabinoside may actually exacerbate the neurotoxicity of IT MTX [12]. Because few studies reported treatment protocol details, we could not examine treatment intensity as a moderator. Research also should examine other treatment modalities that have been implicated in neuropsychological dysfunction, such as corticosteroids (e.g., dexamethasone) [36]. Finally, interventions need to be studied, such as cognitive remediation [37], psychostimulant medication [38], and intensive tutoring [39].

Future research also must use carefully matched comparison groups, as studies may be of limited generalizability if they use 
normative data as a comparison group, newly created measures, or IQ tests only $[2,10]$. It is important to develop a standardized battery within each culture to obtain the best estimate of neuropsychological sequelae in a culturally fair manner. Finally, studies from other cultures may reflect a different medical treatment protocol or some unique aspect of supportive intervention (e.g., more intense tutoring) during cancer treatment. Future studies may benefit from examining supportive academic interventions in more detail as a potential moderator.

These findings inform clinical care, as individualized neuropsychological monitoring and academic intervention (e.g., special education, classroom accommodations) may enhance functional outcomes for ALL survivors including graduation and job attainment rates and long-term adaptive skills to transition to adulthood. A standardized neuropsychological test battery is critical; one has been proposed [40], although widespread implementation (particularly internationally) may be hindered by obstacles such as variable institutional commitment to neuropsychological assessment and insurance reimbursement issues. Finally, baseline testing of all young ALL patients is needed to track neuropsychological and academic skills over time to facilitate early intervention and prevent academic failure.

\section{ACKNOWLEDGMENT}

We wish to thank Jim Spilsbury, PhD, Jonathan Stange, and Shipra Gupta for their research assistance. Preparation of this manuscript was supported in part by a grant from the Lance Armstrong Foundation to the Center for Survivors of Childhood Cancer at Rainbow Babies and Children's Hospital, Cleveland, $\mathrm{OH}$, with which the first and last authors were affiliated when initiating this work.

\section{REFERENCES}

1. Cousens P, Waters B, Said J, et al. Cognitive effects of cranial irradiation in leukemia: A survey and meta-analysis. J Child Psychol Psychiatry 1988;29:839-852.

2. Butler RW, Copeland DR. Neuropsychological effects of central nervous system prophylactic treatment in childhood leukemia: Methodological considerations. J Pediatr Psychol 1993;18:319338.

3. Fletcher JM, Copeland DR. Neurobehavioral effects of central nervous system prophylactic treatment of cancer in children. J Clin Exp Neuropsychol 1988;10:495-538.

4. Brown RT, Madan-Swain A. Cognitive, neuropsychological, and academic sequelae in children with leukemia. J Learn Disabil 1993;26:74-90.

5. Cousens P. Specific learning problems among children treated for acute lymphoblastic leukemia. Int J Pediatr Hematol/Oncol 1997; 4:353-362.

6. Mulhern RK, Butler RW. Neurocognitive sequelae of childhood cancers and their treatment. Pediatr Rehabil 2004;7:1-14.

7. Campbell LK, Scaduto M, Sharp W, et al. A meta-analysis of the neurocognitive sequelae of treatment for childhood acute lymphocytic leukemia. Pediatr Blood Cancer 2006;49:65-73.

8. Moore BD, Copeland DR, Ried H, et al. Neurophysiological basis of cognitive deficits in long-term survivors of childhood cancer. Arch Neurol 1992;49:809-817.

9. Rourke BP. Nonverbal learning disabilities: The syndrome and the model. New York: Guilford; 1989.

10. Moleski M. Neuropsychological, neuroanatomical, and neurophysiological consequences of CNS chemotherapy for acute lymphoblastic leukemia. Arch Clin Neuropsychol 2000;15:603630.

11. Brown RT, Madan-Swain A, Walco GA, et al. Cognitive and academic late effects among children previously treated for acute lymphocytic leukemia receiving chemotherapy as CNS prophylaxis. J Pediatr Psychol 1998;23:333-340.

12. Giralt J, Ortega JJ, Olive T, et al. Long-term neuropsychologic sequelae of childhood leukemia: Comparisons of two CNS prophylactic regimens. Int J Radiat Oncol Biol Phys 1992;24: 49-53.

13. Raymond-Speden E, Tripp G, Lawrence B, et al. Intellectual, neuropsychological, and academic functioning in long-term survivors of leukemia. J Pediatr Psychol 2000;25:59-68.

14. von der Weid N, Mosimann I, Hirt A, et al. Intellectual outcome in children and adolescents with acute lymphoblastic leukaemia treated with chemotherapy alone: Age- and sex-related differences. Eur J Cancer 2003;39:359-365.

15. Brown RT, Sawyer MB, Antoniou G, et al. A 3-year follow-up of the intellectual and academic functioning of children receiving central nervous system prophylactic chemotherapy for leukemia. J Dev Behav Pediatr 1996;17:392-398.

16. Kaemingk KL, Carey ME, Moore IM, et al. Math weaknesses in survivors of acute lymphoblastic leukemia compared to healthy children. Child Neuropsychol 2004;10:14-23.

17. Hill DE, Ciesielski KT, Sethre-Hofstad L, et al. Visual and verbal short-term memory deficits in childhood leukemia survivors after intrathecal chemotherapy. J Pediatr Psychol 1997;22:861-870.

18. Lesnick PG, Ciesielski KT, Hart BL, et al. Evidence for cerebellarfrontal subsystem changes in children treated with intrathecal chemotherapy for leukemia: Enhanced data analysis using an effect size model. Arch Neurol 1998;55:1561-1568.

19. Precourt S, Robaey P, Lamothe I, et al. Verbal cognitive functioning and learning in girls treated for acute lymphoblastic leukemia by chemotherapy with or without cranial irradiation. Dev Neuropsychol 2002;21:173-195.

20. Schatz J, Kramer JH, Ablin AR, et al. Visual attention in long-term survivors of leukemia receiving cranial radiation therapy. J Int Neuropsychol Soc 2004;10:211-220.

21. Rodgers J, Marckus R, Kearns P, et al. Attentional ability among survivors of leukaemia treated without cranial irradiation. Arch Dis Child 2003;88:147-150.

22. Kingma A, Van Dommelen RI, Mooyaart EL, et al. No major cognitive impairment in young children with acute lymphoblastic leukemia using chemotherapy only: A prospective longitudinal study. J Pediatr Hematol Oncol 2002;24:106-114.

23. Kingma A, van Dommelen RI, Mooyaart EL, et al. Slight cognitive impairment and magnetic resonance imaging abnormalities but normal school levels in children treated for acute lymphoblastic leukemia with chemotherapy only. J Pediatr 2001;139:413420.

24. Schatz J, Kramer JH, Ablin A, et al. Processing speed, working memory, and IQ: A developmental model of cognitive deficits following cranial radiation therapy. Neuropsychology 2000;14: 189-200.

25. Brown RT, Madan-Swain A, Pais R, et al. Cognitive status of children treated with central nervous system prophylactic chemotherapy for acute lymphocytic leukemia. Arch Clin Neuropsychol 1992;7:481-497.

26. Strauss E, Sherman EMS, Spreen O. A compendium of neuropsychological tests: Administration, norms, and commentary. New York: Oxford University Press; 2006.

27. Jaccard J. Zumastat statistical methods. Miami, FL: Applied Statistical Analysis; 2006.

28. Cohen J. Statistical power analysis for the behavioral sciences, 2nd edition. Hillsdale, NJ: Lawrence Erlbaum Associates; 1988. 
29. Hedges LV, Olkin I. Statistical methods for meta-analysis. Orlando, FL: Academic Press; 1985.

30. Lipsey MW, Wilson DB. Practical meta-analysis. Thousand Oaks: Sage Publications; 2001.

31. Waber DP, Gioia G, Paccia J, et al. Sex differences in cognitive processing in children treated with CNS prophylaxis for acute lymphoblastic leukemia. J Pediatr Psychol 1990;15:105-122.

32. Wechsler D. Manual for the Wechsler Intelligence Scale for children-Revised. San Antonio, TX: Psychological Corporation; 1974.

33. Wechsler D. Manual for the Wechsler Intelligence Scale for children, 3rd edition. San Antonio, TX: Psychological Corporation; 1991.

34. Wechsler D. Wechsler Adult Intelligence Scale-Revised manual. San Antonio, TX: Psychological Corporation; 1981.

35. Wechsler D. Wechsler Adult Intelligence Scale, 3rd edition: Administration and Scoring Manual. San Antonio, TX: Psychological Corporation; 1997.
36. Waber DP, Carpentieri SC, Klar N, et al. Cognitive sequelae in children treated for acute lymphoblastic leukemia with dexamethasone or prednisone. J Pediatr Hematol Oncol 2000;22:206-213.

37. Butler RW, Copeland DR. Attentional processes and their remediation in children treated for cancer: A literature review and the development of a therapeutic approach. J Int Neuropsychol Soc 2002;8:115-124.

38. Mulhern RK, Khan RB, Kaplan S, et al. Short-term efficacy of methylphenidate: A randomized, double-blind, placebo-controlled trial among survivors of childhood cancer. J Clin Oncol 2004; 22:4795-4803.

39. Moore IM, Espy KA, Kaufmann P, et al. Cognitive consequences and central nervous system injury following treatment for childhood leukemia. Semin Oncol Nurs 2000;16:279-290.

40. Mulhern RK, Butler RW. Neuropsychological late effects. In: Brown R, editor. Comprehensive handbook of childhood cancer and sickle cell disease: A biopsychosocial approach. New York: Oxford University Press; 2006. 